\title{
EL APRENDIZAJE DEL ÁLGEBRA ESCOLAR DESDE UNA PERSPECTIVA PSICOLÓGICA
}

KIERAN, C. ${ }^{(1)}$ y FILLOY YAGÜE, E. (2)

(1) Université de Québec. Montréal, Canadá.

(2) Centro de Investigación y Estudios Avanzados del IPN, México. University of London, Institute of Education. Inglaterra.

Traducción castellana de Luis Puig.

\section{SUMMARY}

This paper describes some of the main contributions of research to the growing knowledge of the cognitive processes which the learning of algebra in secondary schools involves. The continuous attempts of researchers to develop a theory on the teaching/learning of algebra are also discussed. Finally, some future trends in the teaching/learning of algebra are mentioned.

Hace doce años, en el ICME3 en Karlsruhe, Bauersfeld y Skowronek (1976) presentaron un informe titulado "Investigación relacionada con el proceso de aprendizaje de las matemáticas". Ese informe y la discusión que le siguió señaló un cambio significativo en la dirección emprendida por la investigación en educa* ción matemática. La desilusión con los resultados de la investigación conductista previa y con la teoría conductista - producida por su fracaso en dar cuenta de los procesos de aprendizaje en sí mismos-impulsaron a los autores del informe a sugerir que "no deberíamos comenzar desde una teoría del aprendizaje general y neutral respecto del contenido, y derivar de ella una teoría del aprendizaje matemático..., [más bien deberíamos] empezar [desde] procesos de aprendizaje específicos de un contenido" (Bauersfeld y Skowronek 1976, p. 244). Este énfasis que sugirieron en los procesos de aprendizaje específicos de un contenido ha caracterizado la mayor parte de la investigación en álgebra realizada durante los últimos doce años. Una de las intenciones de este artículo es describir algunas de las contribuciones principales de la investigación a un cuerpo creciente de conocimientos sobre los procesos cognitivos involucrados en el aprendizaje del álgebra de secundaria. Este artículo discute también los intentos continuados de los investigadores de desarrollar una teoría de la enseñanza/aprendizaje del álgebra. Finalmente, el artículo concluye con algunas tendencias futuras en el aprendizaje y la enseñanza del álgebra escolar.

\section{PROCESOS COGNITIVOS INVOLUCRA- DOS EN EL APRENDIZAJE DE ALGEBRA DE SECUNDARIA}

Ya que no es posible discutir la investigación reciente en álgebra en su totalidad en un artículo de la extensión de éste, hemos decidido centrarnos en algunos de los temas principales que han sido investigados: el marco de referencia aritmético; variables, expresiones y ecuaciones; resolución de ecuaciones; funciones y sus gráficas; enfoques que usan computadoras. Muchos de los estudios que van a ser referenciados son los que han sido llevados a cabo por investigadores en álgebra del Internacional Group of the Psychology of Mathematics Education (PME) -un grupo que se formó en el congreso del ICME mencionado antes.

\section{El marco artimético de referencia}

Los adolescentes, al comenzar el estudio del álgebra, traen consigo las nociones y los enfoques que usaban en aritmética. Sin embargo, el álgebra no es simplemente una generalización de Ia aritmética. Aprender álgebra no es meramente hacer explícito lo que estaba implícito en la aritmética. El álgebra requiere un cambio en el pensamiento del estudiante de las situaciones numéricas concretas a proposiciones más generales sobre números y operaciones. La transición desde lo 
que puede considerarse como un modo informal de representación y de resolver problemas, a uno formal resulta ser difícil para muchos de los que comienzan a estudiar álgebra. Estos estudiantes siguen usando los métodos que les funcionaban en aritmética. De hecho, un marco de referencia aritmético da cuenta de: a) su forma de ver el signo igual, b) sus dificultades con la concatenación y con algunas de las convenciones de notación del álgebra, y c) su falta de habilidad para expresar formalmente los métodos y los procedimientos que usan para resolver problemas. También da cuenta, en gran medida, de su interpretación de las variables -como se verá en el apartado siguiente.

\section{Forma de ver el signo igual}

La idea extendida entre los estudiantes que comienzan con el álgebra de que el signo igual es la "señal de hacer algo" antes que un símbolo de la equivalencia entre los lados izquierdo y derecho de una ecuación (Kieran 1980) viene indicada por su renuencia inicial a aceptar proposiciones tales como $4+3=6+1$. El pensar que el lado derecho debería indicar el resultado mesto es, $4+3=7$ - les permite dotar de significado a ecuaciones tales como $2 x+3=7$, pero no a ecuaciones tales como $2 x+3=x+4$. El que los estudiantes conciban el signo igual como un mero separador entre la secuencia de operaciones y el resultado les lleva a violar las propiedades simétrica y transitiva de la igualdad. Por ejemplo, al resolver el problema: "Si empiezo la semana con 75 dólares, luego gano otros 24 dólares, y luego gasto 37 dólares, ¿cuántos dólares tendré al final de la semana?", los estudiantes escriben $75+24=99 \quad * 37=62$ (Vergnaud 1984). Esta abreviatura de los pasos se observa también cuando estudiantes mayores resueiven ecuaciones:

$$
\begin{gathered}
2 x+3=5+x \\
2 x+3=5+x-3 \\
2 x=5+x-x-3 \\
2 x-x=5-3 \\
x=2
\end{gathered}
$$

El que estudiantes de álgebra mayores continúan viendo el signo igual como una "señal de hacer algo" y, de hecho, extienden el conjunto de símbolos de operaciones matemáticas para incluir en él el signo igual se comprobó en un estudio con 150 estudiantes de primer ciclo de universidad (Mevarech y Yitschak 1983). Estos mismos estudiantes tuvieron éxito en un $90 \%$ al resolver un conjunto de ecuaciones lineales, lo que indica que una comprensión pobre de la equivalencia y del signo igual no está basada en falta de destreza o falta de familiaridad con las ecuaciones lineales.

\section{Dificultades con las convenciones de notación}

En aritmética, la concatenación denota adición (p.e., 37 significa $30+7 ; 2 \frac{\frac{1}{4}}{4}$ significa $2+\frac{1}{4}$ ). Sin embargo, en álgebra, la concatenación significa multiplicación (p.e., $4 \mathrm{~b}$ significa $4 \times \mathrm{b}$ ). Extender la generalización sobre la base de lo que era correcto en aritmética puede conducir a los alumnos que empiezan con el álgebra a malinterpretar el sentido de los términos algebraicos. Así, se han encontrado estudiantes que interpretan $4 p$ como 42 e incluso como "4 patatas".

Otra convención que los estudiantes parece que no usan en su aritmética escolar elemental es el uso de paréntesis y el orden de las operaciones. Incluso cuando se les introduce al uso de paréntesis en su curso de álgebra, los estudiantes a menudo no consideran que los paréntesis sean necesarios para denotar el orden en que se efectúan las operaciones (Kieran 1979) -el orden de izquierda a derecha en que están escritos los términos especifica para esos estudiantes el orden del cálculo. De la misma manera, la jerarquía convencional de las operaciones parece ser un conjunto innecesario de reglas para los estudiantes que comienzan el álgebra.

No son sólo las convenciones numéricas lo que crea di. ficultades a los novicios en álgebra: tampoco es obvia para ellos la notación que ha de usarse para expresar respuestas algebraicas. Por ejemplo, uno de los items del test CSMS (Concepts in Secondary Mathematics and Science), que se pasó a 2820 estudiantes británicos de secundaria, les pedía que determinaran el área del rectángulo que se muestra en la figura 1 .

figura I

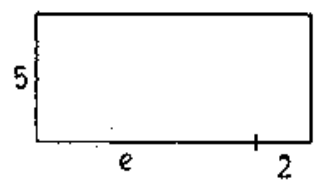

El $42 \%$ de los alumnos de 13 años respondieron $5 \mathrm{e} 2,0$ e 10, o 10e, o e+10 (Küchemann 1981). Este ítem y otros del test CSMS se usaron en el estudio SESM (Strategies and Errors in Secondary Mathematics), una secuela del estudio anterior que también se realizó con alumnos entre 13 y 16 años (Booth 1981). Las entrevis. tas con estudiantes que hicieron los mismos errores de notación que hemos indicado antes indicaron que la habilidad para describir verbalmente un método no trae consigo necesariamente la habilidad para simbolizar ese método matemáticamente. Booth (1983) señaló también que los estudiantes pueden responder correctamente a ftems que requieren el uso de una cierta notación o unas ciertas convenciones y ser incapaces sin embargo de discriminar entre representaciones correctas e incorrectas. Esto sugiere, según Booth, que la comprensión de las notaciones puede avanzar por etapas.

\section{Métodos de simbolizar}

El harto documentado uso de métodos informales por parte de los niños en la escuela elemental les permite resolver problemas sin tener que ser muy específicos sobre los procedimientos que usan. Su confianza en métodos intuitivos no enseñados y el que se centren en conseguir la respuesta va en contra de que presten atención al método que usan. El álgebra les fuerza a 
formalizar procedimientos por los que puede que antes nunca se hayan preocupado.

De hecho, los estudiantes que comienzan con el álgebra no logran darse cuenta de que el procedimiento es a menudo la respuesta. Por ejemplo, el resultado de sumar 5 y b se enuncia como $5+b$. Los estudiantes no sólo deben superar lo que Matz y Davis han llamado el dilema "proceso-producto" y adquirir lo que Collis ha llamado "aceptación de la falta de cierre", sino que también tienen que debilitar sus "expectativas artiméticas acerca de las respuestas bien-formadas, es decir, que una respuesta es un número" (Matz 1980, p. 132).

\section{Variables}

La experiencia de los niños en la escuela elemental con las letras en ecuaciones se reduce a menudo a fórmulas como $\mathrm{A}=\mathrm{bxh}$, y relaciones entre unidades de medida como $10 \mathrm{~mm}=1 \mathrm{~cm}$. La primera supone reemplazar b y h por valores diferentes para encontrar el área de rectángulos dados; la segunda regla se usa para encontrar, por ejemplo, el número đe milímetros a que $\mathrm{co}^{-}$ rresponde 5 centímetros. Este segundo uso de las letras como etiquetas es el que interfiere a menudo con la forma como los estudiantes llegan a entender el significado de los términos variables en las ecuaciones algebraicas. En la segunda "ecuación" de arriba, no sólo se leen las letras como etiquetas, sino que además el signo igual se lee como una preposición: "hay 10 milímetros en 1 centímetro". De hecho, incluso estudiantes mayores malinterpretan el sentido de las variables en las ecuaciones. El $38 \%$ de los 150 alumnos de primer ciclo de universidad examinados por Mevarech y Yitschak (1983) contestaron que, en la ecuación $3 \mathrm{k}=\mathrm{m}$, $\mathrm{k}$ es mayor que $\mathrm{m}$. Si los estudiantes consideraran el signo igual como un símbolo de equivalencia, probablemente serían capaces de evitar el cometer tales errores.

Otras interpretaciones que los estudiantes de álgebra asignan a las letras han sido estudiadas sistemáticamente por Kiichemann (1981) en el proyecto de gran escala CSMS. Usando una clasificación desarrollada originalmente por Collis (1975), Küchemann encontró que la mayoría de los estudiantes trataban las letras en expresiones y ecuaciones como incógnitas específicas más que como números generalizados o como variables. Por ejemplo, el 55\% de los niños de 13 años encuestados afirmaron que $\mathrm{L}+\mathrm{M}+\mathrm{N}=\mathrm{L}+\mathrm{P}+\mathrm{N}$ nunca es verdad. Harper (1981) sugirió la existencia de etapas en la comprensión de un término literal como variable, y señaló que los estudiantes usan los téminos literales mucho antes de que sean capaces de conceptualizarlos como variables -esto es, de percibir lo general en lo particular. En un experimento de enseñanza diseñado específicamente para favorecer la adquisición de la nocion de letra como número generalizado, Booth (1982, 1983) encontró una fuerte resistencia por parte de los alumnos a asimilar esta parte del álgebra. Booth sugiere que "la obtención de este nivel de conceptualización está relacionada con el desarrollo de estructuras cognitivas de orden más alto" (Booth 1984, p. 88).

\section{Expresiones y ecuaciones}

Expresiones. Se diseñó un experimento de enseñanza para superar la incapacidad que los estudiantes tienen de aceptar las expresiones algebraicas como "soluciones de problemas" (Chalouh y Herscovics 1984, Herscovics y Chalouh 1984). En los problemas aparecían disposiciones rectangulares de puntos, líneas divididas en segmentos y áreas de terrenos rectangulares -en todos los problemas, una de las dimensiones estaba oculta. La secuencia de enseñanza permitía a los estudiantes construir significado para expresiones algebraicas tales como $2 x+5 x$. Sin embargo, los estudiantes creían que estas expresiones estaban incompletas en algún sentido. Se sentían obligados a expresarlas como parte de una igualdad, tal como Área $=2 x+5 x$ o como $2 x+5 x=$ algo. En otro estudio (Kieran 1983) se encontró que algunos de los estudiantes no podían asignar significado alguno a $a$ en la expresión $a+3$ porque la expresión carecía de un signo igual y un miembro de la derecha.

Ecuaciones. En la escuela elemental, los niños "resuel. ven" ecuaciones sencillas como $3+\square=8$ o $3+n=8-$ que a veces se llaman proposiciones de "sumando faltante". Sin embargo, estas ecuaciones se presentan a menudo fuera del contex to de auténticas situaciones de problemas verbales, con el resultado de que el niño carece de un apoyo en el "mundo real" para interpretarlas. De hecho, los niños casi nunca usan ecuaciones para representar los problemas aritméticos verbales y, si se les pide una ecuación, los niños resuelven primero el problema y luego intentan dar la ecuación. A menudo los niños que son capaces de resolver problemas verbales no pueden escribir las ecuaciones que representan las relaciones cualitativas de la situación del problema. Cuando escriben una ecuación, ésta representa por regla general las operaciones que habían usado para resolver el problema, no contiene una incógnita y el resultado del cálculo está usualmente en el lado derecho del signo igual.

La percepción que los niños tienen del significado de las proposiciones de sumando desconocido no ha sido investigada, que nosotros sepamos. Sabemos, sin embargo, que los procesos que usan los niños para resolver las proposiciones de sumando desconocido incluyen "contar hacia adelante", "contar hacia atrás", "substitución" y "uso de hechos numéricos conocidos" (Booth 1987, Nesher 1980). Presumimos que las concepciones primitivas de los niños de lo que es una ecuación no contienen, en general, la idea de que tengan términos literales a ambos lados del signo igual. Las ecuaciones de ese estilo carecen probablemente de sentido, a la vista de la presunta concepción ingenua de los niños de una ecuación como un hecho numérico ligeramente disfrazado con la falta de algún componente. La concepción de que "una ecuación es una representación de una relación numérica en la que el lado izquierdo tiene el mismo valor que el lado derecho" fue objeto de un experimento de enseñanza con niños de 12 y 13 años (Herscovics y Kieran 1980, Kieran 1981). Ese estudio mostró que es posible cambiar la percepción de las ecuaciones que tienen los estudiantes que 
comienzan el álgebra como algo unidireccional y con la respuesta en el lado derecho.

\section{Resolución de ecuaciones}

Muchas investigaciones sobre álgebra hechas en el marco del PME se han centrado en la manera como los estudiantes enfocan la resolución de ecuaciones. Los enfoques usados se pueden clasificar en tres tipos: a) intuitivo, b) sustitución por tanteo, y c) formal.

Los enfoques de resolución intuitivos incluyen el uso de hechos numéricos, técnicas de recuento, y métodos de recubrimiento. Por ejemplo, resolver $5+n=8$ trayendo a colación el hecho numérico aditivo de que $5+3$ es 8 sería un uso de hechos numéricos conocidos. Resolver la misma ecuación contando $5,6,7,8$ y dándose cuenta de que se nombraron tres números después del 5 para llegar a 8 sería un ejemplo de resolución por técnicas de recuento. Booth (1983) ha señalado el uso de ambos métodos entre estudiantes novicios de álgebra. Bell, O'Brien y Shiu (1980) han visto alumnos que usaban un método de "recubrimiento" para resolver ecuaciones tales como $2 x+9=5 x$ : "Ya que $2 x+9$ vale $5 x$, el 9 debe ser lo mismo que $3 x$ porque $2 x+3 x$ también es igual a 5x; así que $x$ es 3 ". Petitto (1979) señaló que las técnicas intuitivas a menudo no se generalizan -como en las ecuaciones en que aparecen números negativos-, y observó que los estudiantes que usaban una combinación de procesos formales e intuitivos tuvieron más éxito que los que usaron uno solo de esos procesos.

El uso de substitución por tanteo como un método de resolución de ecuaciones (p.e., resolver $2 x+5=13$ probando valores diferentes como $2,3,5$ y 4) consume mucho tiempo y coloca una carga pesada en la memoria de trabajo, excepto si todos los intentos se anotan de algún modo. Tan pronto como los estudiantes de álgebra aprenden a manejar un método formal de resolución de ecuaciones, tienden a abandonar el uso de la substitución (Kieran 1985). Desgraciadamente, parece que también lo abandonan como un mecanismo para verificar la corrección de su solución (Lewis 1980). Sin embargo, hay pruebas de que los estudiantes que usan la substitución como un mecanismo primerizo de resolucion de ecuaciones -y no todos lo hacen- poseen una noción más desarrollada del equilibrio entre los lados izquierdo y derecho de una ecuación y del papel del signo igual como equivalencia, que la que poseen los estudiantes que nunca usan la substitución como un método de resolver ecuaciones (Kieran 1988).

Los métodos formales de resolución de ecuaciones incluyen la transposición de términos (esto es, "cambiar de lado -cambiar de signo") y ejecutar la misma operación en ambos lados de la ecuación. Aunque la transposición esté considerada por muchos profesores de álgebra como una versión abreviada del procedimiento de realizar la misma operación en ambos lados, los estudiantes que empiezan con el álgebra parece que perciben de forma bastante diferente esos dos métodos de resolución de ecuaciones (Kieran 1988). El procedi* miento de ejecutar la misma operación en los dos lados de una ecuación pone el énfasis en la simetría de una ecuación; este énfasis está ausente en el procedimiento de transposición. En un experimento de enseñanza diseñado para ayudar a los estudiantes a construir significado para el procedimiento de ejecutar la misma operación en los dos lados de la ecuación (Kieran 1983), se encontró que los estudiantes que habían empezado el estudio teniendo preferencia por el método de transposición no fueron capaces, en general, de dotar de sentido al procedimiento que se les estaba enseñando. La secuencia de instrucción pareció tener su mayor impacto sobre aquellos estudiantes que habían empezado el estudio con una preferencia inicial por el método de substitución y que veían la ecuación como una balanza entre los lados izquierdo y derecho.

Filloy y Rojano (1985a, 1985b) han usado también modelos concretos en sus experimentos de enseñanza de resolución de ecuaciones. En su informe indican que muchos estudiantes tendían a anclarse en los modelos y parecían incapaces de ver los lazos entre las operaciones que ejecutaban en el modelo y las operaciones algebraicas correspondientes. Como resultado de ello, los estudiantes permanecían dependientes del modelo incluso cuando éste ya no era útil. De hecho los estudiantes intentaban usar el modelo para ecuaciones sencillas que podían haber sido resueltas, más fácilmente, mediante los métodos intuitivos de resolución de ecuaciones que habían usado antes de que se les enseñara el nuevo método. Estaban hasta tal punto anclados en los procesos desarrollados en el modelo concre to que se les había enseñado, que parecían olvidar los métodos que usaban previamente.

Algunos otros estudios han encarado el asunto del conocimiento de los estudiantes de la estructura de las ecuaciones y la resolución de ecuaciones (Kieran, en prensa). Wagner, Rachlin y Jensen (1984) encontraron que los estudiantes de álgebra tienen dificultad en tratar expresiones con muchos términos como una sola unidad y no perciben que la estructura superficial de $4(2 r+1)+7=35$, por ejemplo, es la misma que la de $4 x+7=35$.

Otro aspecto estructural que los estudiantes que empiezan con el álgebra se supone que han de aprender concierne a la relación entre las operaciones y sus inversas y las expresiones equivalentes de esas relaciones. Asumimos que los estudiantes que entran en los primeros años de secundaria, hacia los doce años, saben por ejemplo que $3+4=7$ puede expresarse como $3=7-4$, y que serán capaces de generalizar este conocimiento a ecuaciones que comportan términos literales, llegando a ser conscientes por ello de que $x+4=7$ y $x=7-4$ son equivalentes y tienen, por tanto, la misma solución. Ahora bien, dos errores que cometen los aprendices de álgebra muestran que les es difícil juzgar las expresiones equivalentes de la relación adición/ substracción (Kieran 1984): en el error "intercambio de sumandos", se juzga que $x+37=150$ tiene la misma solución que $x=37+150$; en el error "redistribución", se juzga que $x+37=150$ tiene la misma solución que $x+37-10=150+10$. 
Greeno (1982) ha señalado que los estudiantes que empiezan en álgebra no son consistentes en la manera como dividen las expresiones algebraicas en sus partes constitutivas. Por ejemplo, pueden simplificar $4(6 x-3 y)+5 x$ como $4(6 x-3 y+5 x)$ en una ocasión, pero hacer algo distinto en otra ocasion. Un cambio en el contex to de la tarea puede conducir a una estructuración diferente de la expresión (Chaiklin y Lesgold 1984).

Un estudio reciente con una componente de enseñanza ha mostrado que la instrucción puede mejorar la habilidad de los estudiantes para reconocer la forma o estructura superficial de una ecuación algebraica. Thompson y Thompson (1987) diseñaron un experimento de enseñanza que contenía dos formatos de instrucción: a) notación de ecuaciones algebraicas, y b) árboles de expresiones presentados en la pantalla de una computadora. Después de la instrucción, 8 estudiantes de séptimo no generalizaron las reglas más allá de su campo de aplicación, ni dejaron de percibir la estructura de las expresiones. Los estudiantes desarrollaron además una noción general de variable como "lugar para rellenar" dentro de una estructura y la opinión de que una variable puede ser reemplazada por cualquier cosa -un número, otra letra o una expresión.

Otro aspecto de conocimiento estructural que se considera importante en la resolución de ecuaciones supone el conocimiento de restricciones de equivalencia. Greeno (1982) ha señalado que los novicios en álgebra carecen del conocimiento de las restricciones que determinan si las transformaciones están permitidas. Por ejemplo, no saben cómo mostrar que una solución incorrecta está mal obtenida, excepto volviendo a resolver la ecuación dada. No parecen ser conscientes de que una solución incorrecta, si se substituye en la ecuación inicial, da origen a valores diferentes para el lado izquierdo y el lado derecho de la ecuación. Ni tampoco se dan cuenta de que sólo la solución correcta da origen a valores equivalentes para las dos expresiones en cualquiera de las ecuaciones de la cadena que resuelve la ecuación. Sin embargo, no son sólo los resolutores de ecuaciones novicios los que carecen del conocimiento de estas restricciones de equivalencia. Kieran (1984) encontró que también carecían đe este conocimiento los resolutores de ecuaciones experimentados y competentes de la enseñanza secundaria obligatoria.

\section{Funciones y sus gráficas}

La siguiente área principal de investigación sobre el aprendizaje đel álgebra que vamos a discutir se refiere a las funciones y sus gráficas. Dreyfus y Eisenberg (1981) investigaron las bases intuitivas de los conceptos relacionados con las funciones entre 440 estudiantes de sexto a noveno grado (equivalentes a $6^{\circ}$ de EGB hasta $1^{\circ}$ de BUP). Hicieron preguntas sobre imagen, antiimagen, crecimiento, valores extremos, y pendientes en tres formatos de representación -gráficas, diagramas y tablas de pares ordenados-tanto en contextos abstractos como concretos. Se encontró que los estudiantes más capaces preferían el formato gráfico para todos los conceptos, mientras que los estudiantes menos capaces preferían el formato de tablas. Didácticamen- te, esto sugiere que los subconceptos de función deberían introducirse en formato de gráfica para los estudiantes de alto nivel y en formato de tablas para los estudiantes de bajo nivel.

Las dificultades que experimentan muchos estudiantes de álgebra en comprender el significado de las representaciones gráficas de las funciones ha sido ilustrada en varias investigaciones (p.e. Clement 1985, Javier 1981, Kerslak 1977, Ponte 1985). Se ha encontrado que muchos estudiantes tienen problemas en establecer la conexión entre los datos numéricos y los datos gráficos que involucra el plano cartesiano. Se han identificado dificultades similares con respecto a la recta numérica, especialmente al tratar con escalas (Vergnaud y Errecalde 1980).

Markovits, Eylon y Bruckheimer (1983) examinaron alumnos de noveno grado (equivalente a $1^{\circ}$ de BUP) que habían sido introducidos al concepto de función como una correspondencia de varios a uno entre un dominio y un rango. En la primera parte del estudio, se les pidio a los estudiantes que dieran ejemplos de funciones que satisfacieran algunas restricciones determinadas y que especificaran cuántas funciones de ese estilo existen. En la segunda parte, los investigadores examinaron el efecto del contexto (matemático vs científico). Encontraron que, independientemente del contex to, la concepción de los estudiantes de la función era lineal. La mayoría de las funciones que dibujaron los estudiantes se componían de segmentos rectos. Los estudiantes más capaces tuvieron mejores resultados en el contexto puramente matemático que en el contexto científico; los estudiantes menos capaces tuvieron mejores resultados en el contexto científico.

En otro estudio que involucraba a 60 estudiantes de 16 a 18 años que ya estaban familiarizados con la noción de función y con su definición estructural formal, Sfard (1987) intentó determinar si esos estudiantes concebían las funciones operativamente más que estructuralmente. Una concepción operativa es la que ve una función como un algoritmo para calcular una magnitud cambiante por medio de otra. Una concepción estructural es la que ve una función como una correspondencia entre dos conjuntos. La mayoría de los alumnos que fueron examinados concebían las funciones como un proceso más que como un constructo estático. En una segunda fase del estudio que involucraba a 96 estudiantes de 14 a 17 años, se les pidió que tradujeran cuatro problemas verbales sencillos a ecuaciones y también que proporcionaran prescripciones verbales (algoritmos) para calcular las soluciones de problemas similares. Tuvieron mucho más éxito con las prescripciones verbales que con la construcción de ecuaciones. Estos resultados apoyan los de un estudio previo (Soloway, Lochhead y Clement 1982) que mostró que los estudiantes se las arreglan bien para traducir un problema verbal a una "ecuación" cuando esa ecuación tiene la forma de un programa corto de computadora que especifica cómo encontrar la solución. Estos resultados pueden verse como evidencia del predominio de las concepciones operacionales entre nuestros estudiantes de álgebra. 
Los resultados del estudio de Sfard plantean algunas preguntas importantes que atañen a la enseñanza de las matemáticas. Los símbolos y las definiciones que se enseñan en la escuela son claramente estructurales, no operacionales -en el sentido de Sfard. Este enfoque tradicional de la enseñanza del álgebra no parece ser muy eficaz. Según Sfard, "si una concepción operacional es verdaderamente el primer escalón necesario en la adquisición de una idea matemática nueva, podemos probablemente precipitar el aprendizaje favoreciendo la comprensión por parte de los estudiantes de los procesos y algoritmos, antes de traducirlos a definiciones estructurales; esto puede hacerse incorporando la programación de computadoras en los cursos de matemáticas" (Sfard 1987, p. 168). La sugerencia de Sfard respecto a la integración de enfoques mediante computadoras en la enseñanza del álgebra se refleja en varios estudios recientes presentados en reuniones del PME.

\section{Enfoques mediante computadoras}

La computadora ofrece una gama de oportunidades de representación para los conceptos de las matemáticas escolares. Hay un dominio conceptual, en particular, que parecería especialmente adecuado para el potencial de representación dinámica que proporcionan las microcomputadoras: se trata de las gráficas de funciones. Antes de examinar el cuerpo de investigación relacionado con este uso de las computadoras, primero echaremos una ojeada a los estudios que han investigado el aprendizaje, cuando se usan computadoras, del concepto de variable y la identificación de puntos en el espacio mediante números.

Samurcay (1985) investigó el concepto de variable que desarrollan los estudiantes de 9 a 16 años en los distintos entornos de Logo, Pascal y LSE. Encontró que eI concepto de variable no aparece espontáneamente en los alumnos jóvenes que trabajan en un entorno Logo: hace falta una intervención didáctica. El proyecto Logo Math (Hoyles, Sutherland y Evans 1985) incorporó la intervención del profesor en su estudio sobre cómo los estudiantes de 11 a 14 años desarrollan el concepto de variable en un contexto Logo. Sutherland y Hoyles (1986) encontraron que Ios alumnos necesitan experimentar las variables en muchas situaciones diferentes antes de que pueda tener Iugar una síntesis. Al final de los tres años del estudio, se entrevistó a los estudiantes para ver si habían sido capaces de establecer lazos entre el uso de variables en Logo y en álgebra. Algunas de las preguntas de las entrevistas se tomaron del cuestionario CSMS, mencionado antes en este artículo. Sutherland (1987) señaló que la experiencia de Ios alumnos con Logo enriqueció su comprensión de Ias variables en un contexto algebraico. Thomas y Tall (1986) también dieron cuenta de resultados beneficiosos similares, obtenidos en su investigación del aprendizaje del concepto de variable por parte de estudiantes de 12 años en un entorno BASIC.

Algunos otros estudios con una componente principal de uso de computadoras se han centrado en la comprension de los estudiantes de la identificación de puntos en la recta numérica y en el plano cartesiano. Rogalski (1985) enfrentó a estudiantes de 11 a 13 años con un juego de tiro al blanco que Ies exigía que dieran uno o dos números que correspondieran a la posición del blanco. La respuesta en la pantalla tomaba la forma de un rastro dibujado en la pantalla gráfica, $o$ un mensaje si los números dados eran demasiado grandes. Se encontró que los estudiantes eran capaces de tener éxito meramente mediante estrategias de aproximación y no tuvieron que usar la proporcionalidad -algo que Rogalski había esperado encontrar. El interés de este estudio reside en el hecho de que los estudiantes fueron capaces de desarrollar una comprensión de escalas e intervalos a partir de lo que ocurría en la pantalla. Parece más difícil que se adquiera esta conciencia de lo que ocurre en un entorno sin computadoras (Vergnaud y Errecalde 1980).

Actualmente parece menos obvio que sean evidentes los efectos beneficiosos del uso de las computadoras para el aprendizaje de las funciones y las gráficas de funciones. Dreyfus y Eisenberg (1987) usaron el programa de computadora Green Globs (Dugdale 1982) con dos grupos de alumnos de undécimo y duodécimo grados (equivalente a $3^{\circ}$ de BUP y COU) en un estudio diseñado para investigar si este tipo de software facilita a los estudiantes la comprensión de la relación entre las representaciones simbólica y gráfica de una función. Se encontró que el lazo entre las dos representaciones permanecía vago para más de la mitad de los estudiantes. Pruebas adicionales de Ia dificultad de los estudiantes en ligar las representaciones algebraica y geométrica provienen de un estudio del que da cuenta Goldenberg (1987). Usando software que liga dinámicamente representaciones gráficas y simbólicas de funciones, Goldenberg encontró que "ilusiones perceptivas y cambios de atención de un rasgo a otro obscurecen algo de lo que el uso educativo de las gráficas se supone que ha de elucidar" (p. 197).

La investigación que acabamos de discutir da Iugar a alguna preocupación sobre la eficacia de ciertos aspectos del software con representaciones múltiples. Como ha señalado Goldenberg (1987):

"El sentido común apoya la idea de que el uso de más de una representación de las funciones ayudará a los aprendices a entender lo que queda menos claro cuando se usa solo una representación. Presentadas meditadamente, representaciones múltiples y ligadas aumentan la redundancia y pueden reducir así las ambiguiedades que podrían ser inherentes a una representación única. Las expresiones algebraicas especifican la relación exacta, pero no dan ni ejemplos particulares ni una gestalt visual. Las gráficas proporcionan una gestalt dentro de los límites de la gráfica, pero dejan poco claros los detalles menudos. Las tablas proporcionan ejemplos de la aplicación función, pero no especifican su naturaleza. Dicho de otra manera, cada representación bien escogida ve una función desde una perspectiva particular que captura bien algún aspecto de la función pero deja otros menos claros. Múltiples representaciones, tomadas conjuntamente, deberían mejorar la fidelidad de la toralidad del mensaje. Estos argumen- 
tos teóricos son bastante razonables, pero puede que no sean válidos (p. 197).

Otra investigación apoya esta preocupación. Las observaciones de Tall (1987) usando gráficas en computadora con alumnos mayores sugieren que hay indicaciones claras de obstáculos conceptuales que necesitan ser investigados.

La síntesis precedente de algunos de los temas principales de la investigación en la enseñanza/aprendizaje del álgebra desarrollada en esta década no ha sido capaz, por desgracia, de incluir la mención de todos los estudios que se han realizado. Confiamos, sin embargo, en que, al centrarnos en algunas de las cuestiones más globales, hayamos sido capaces de proporcionar una impresión general del tipo de investigaciones que se han emprendido y de lo que se ha obtenido como resultados. Nuestras sugerencias para áreas de investigacion futura potencialmente fructíferas se presentan en la sección de conclusiones de este artículo.

\section{ALGUNAS CONSIDERACIONES TEÓRICAS}

\section{Introducción}

En la mayor parte de la investigación en álgebra que se ha desarrollado recientemente, hay una falta de modelos teóricos paradigmáticos -incluso si usamos el término paradigma (más o menos en el sentido de Kuhn, 1962) no como un sinónimo de teoría, sino, en un sentido más general, como el conjunto de supuestos de base que uno hace sobre la naturaleza y los límites del objeto de estudio propio, el método para estudiarlo, y la decisión sobre qué se toma como evidencia. Tampoco hay consenso sobre cuál de esos supuestos de base debería determinar la forma que toman los marcos teóricos locales para interpretar fenómenos específicos y para proponer nuevos diseños experimentales que hagan avanzar la teoría más lejos con el fin de englobar otras evidencias o nuevas evidencias no relacionadas. En resumen, todavía es necesario hablar acerca de las fronteras de muchos de los proyectos de investigación.

Para empezar, hay algunas otras disciplinas que ya han emprendido investigaciones sobre los mismos asuntos que impregnan la mayoría del trabajo del que han dado cuenta los educadores matemáticos: la lingǘistica, Ia logica, la psicolingüística, la semiótica, la psicología general cognitiva, la psicología de las matemáticas, la epistemología de las matemáticas, la historia de las matemáticas, la psicología de la educación, la teoría del desarrollo del currículo de matemáticas, $\mathrm{y}$, más que ninguna otra, Ia didáctica de las matemáticas. (Usaremos aquí el término didáctica en el sentido en que lo usa la escuela francesa-que hablan de "Didactique des mathématiques". Prestaremos atención por tanto sólo a los fenómenos didácticos cuyas causas puedan atribuirse a la materia matemática implicada en el proceso de enseñanza/aprendizaje -en nuestro caso el uso del "Ienguaje algebraico").

Gran parte de los trabajos de investigación en álgebra han ido incorporando recientemente los resultados de estas disciplinas y han ido redefiniendo esos resultados en el interior de sus propios marcos teóricos. En esta sección del artículo intentamos interpretar intentos teóricos recientes y diferentes de reorganizar lo que ha sido investigado de los procesos de enseñanza/aprendizaje del álgebra durante los últimos cinco años. Tendremos que trabajar con un buen montón de terminología nueva con el fin de articular un discurso que pueda rendir cuenta fielmente de Ia riqueza de la investigación reciente en álgebra. Para minimizar las difícultades del lector, nos limitaremos a usar sólo los términos y conceptos desarrollados en los artículos sobre álgebra del volumen I de Ias actas de Ia Undécima Conferencia Internacional de Psicología de la Educación Matemática (Bergeron, Herscovics y Kieran 1987), y Ios que introduce Eco (1979) en su primer capítulo. Para conseguir nuestro objetivo introduciremos el concepto metodológico de modelo teórico local en el que el objeto de estudio se enfoca desde tres componentes interrelacionadas: a) modelos de enseñanza del álgebra, b) modelos para los procesos cognitivos, y c) modelos de competencia formal.

\section{Nuevas tendencias e influencias correlacionadas}

En primer Iugar, señalaremos que tanto la lingiística, como la teoría del procesamiento de la información y la diđáctica de las matemáticas (Brousseau 1986) han hecho un trabajo importante sobre la noción de código. Esta noción está emergiendo como un concepto clave para interpretar lo que resulta de usar la idea de representación en los modelos explicativos nuevos de los problemas cognitivos que plantean los enfoques de enseñanza alternativos (Janvier 1987) o los medios electrónicos (Kapụt 1987a).

Por poner otro ejemplo, tómese el énfasis que la psicoIingǘstica y la inteligencia artificial ponen en un modelo procesual de las habilidades humanas y relaciónese con la manera como el modelo explica cóno y por qué los usuarios del lenguaje algebraico cometen de forma natural y común errores en sus procedimientos sintácticos (Matz 1982, Kirshne 1987, Thompson y Thompson 1987).

A estos desarrollos ha de añadirse la atención que un punto de vista pragmático ha dado al significado en el uso (Booker 1987, Booth 1987, Filloy 1987, Herscovics y Chalouh 1984, Kieran 1979, Lee y Wheeler 1987, Nunes Carraher y Schliemann 1987, Gallardo y Rojano 1987) con preferencia al significado en abstracto. Por acumulación, estos enfoques, y otros de la misma naturaleza (Putman, Lesgold, Resnick y Sterrett 1987, Vergnaud y Cortés 1986), han conducido a un cambio de dirección en el interior del trabajo reciente en álgebra que se aparta de la "competencia" y va hacia la "actuación" deI usuario del lenguaje algebraico. Este cambio de punto de vista tiene implicaciones fundamentales para la manera como uno mira el lenguaje algebraico. En esencia, la pretensión es que la gramá tica -el sistema formal abstracto del álgebra (como puede encontrarse en los artículos de Kaput, Kirshner y Matz) - y la pragmática-los principios del uso del 
lenguaje algebraico-son dominios complementarios en el estudio de la psicología del aprendizaje del algebra. Ambos son dominios relacionados con los diferentes modelos de enseñanza, innovadores o tradicionales, que se usan para conseguir el objetivo de guiar a los alumnos a que se conviertan en usuarios competentes del álgebra elemental.

\section{Componentes de los modelos teóricos locales}

Las consecuencias de este punto de vista incluyen no sólo una afirmación del lugar central de los asuntos de gramática formal, sino también el reconocimiento de que éstos deben ser encajados en un marco más global que combine explicaciones funcionales $y$ formales. Más aún, para dar cuenta del significado completo de algunos mensajes matemáticos que aparecen durante procesos normales de enseñanza/aprendizaje, al Lado del significado estricto del texto matemático en cuestión, como se hace en Kaput (1987a), hay que admitir algunos otros significados de ciertos otros mensajes (lógicos) que no son emitidos explícitamente por el emisor ni por el receptor: los así llamados supuestos implícitos, o las consecuencias inmediatas, o las implicaciones -todo esto necesita la incorporación de alguna "lógica natural" que considere la relación entre todos estos significados.

Además, siguiendo este mismo curso de ideas, estamos forzados a distinguir entre la competencia en descodificar un mensaje y la competencia en emitir el mismo mensaje. Nuestro enfoque teórico debe tomar en cuenta estos dos tipos de actividades: la producción de mensajes matemáticos y su recepción.

Observaciones empíricas sobre cómo se usan sistemas de signos durante los intercambios de mensajes en el interior de procesos de enseñanza/aprendizaje matemáticos, y las situaciones correspondientes en las que un sujeto usa estos sistemas de signos matemáticos en una situación de resolución de problemas, muestran que los procesos cognitivos implicados entremezclan el nivel de competencia con el nivel pragmático, lo que puede producirse por causas de tipos bastante diferentes.

Hay una componente pragmática que procede del entorno de enseñanza en el que se está llevando a cabo el proceso de aprendizaje. Esta componente está ligada a muchos contratos socialmente institucionalizados, que incluyen no sólo los usos y las formas tradicionales en las que se emiten los mensajes de sistemas de signos en los sistemas educativos, sino también -y con mayor importancia- la presencia usualmente ignorada de toda la evolución histórica de estos sistemas de signos matemáticos -siendo la notación algebraica la manera más inmediata, pero no la más fuerte, de todas las formas particulares de usar los sistemas de signos matemáticos en su aplicación a la ciencia, la tecnología y los procesos de información social actuales.

Mano a mano con todas estas tendencias, hay una componente pragmática que se debe a las estructuras cog- nitivas del sujeto individual que aparece en cada etapa de desarrollo y que da preferencia a mecanismos de procedimiento distintos, formas distintas de codificar y decodificar los mensajes matemáticos pertinentes a la etapa en cuestión, estrategias diferentes de resolución de problemas, etc. Piénsese, por ejemplo, en todas Jas evidencias que hemos acumulado sobre la tendencia de los sujetos a mantener interpretaciones aritméticas de la mayoría de las situaciones algebraicas, incluso en etapas bien avanzadas del estudio del álgebra.

La estabilidad de estos fenómenos y la replicabilidad harto establecida de los diseños experimentales que se han usado para su estudio no nos permite descuidar la consideración de tres componentes importantes en cualquier modelo teórico y nos enfrenta con la necesidad de proponer componentes teóricas que traten con: a) modelos de enseñanza del álgebra (como se intenta en Janvier, 1987, para los números racionales, o en Filloy, 1987, y Gallardo y Rojano, 1987, para la resoIución de ecuaciones lineales), junto con b) modelos de los procesos cognitivos implicados (como se intenta en Goldin 1987, para la resolución de problemas), ambos relacionados con c) modelos de competencia formal que simulen la ejecución competente de un usuario ideal del lenguaje del álgebra elemental (como en los intentos de Kirshner, Matz y Thompson).

Así que, en vez de argüir en favor de privilegiar una cualquiera de esas componentes -gramática, lógica, matemáticas, modelos de enseñanza, modelos cognitivos y pragmática - tendremos que concentrarnos en modelos teóricos locales adecuados sólo a fenómenos específicos, pero capaces de tomar en consideración todas esas componentes, y por tanto proponemos diseños exprimentales ad hoc que arrojen luz sobre las interrelaciones y las oposiciones que ocurren durante la evolución de todos los procesos pertinentes relacionados con cada una de esas tres componentes.

\section{Sistemas matemáticos de signos}

Necesitaremos una noción de "sistemas matemáticos de signos" (que en adelante abreviaremos SMS) suficientemente amplia para que cumpla las tareas recién enumeradas, y una noción de significado de un signo que abarque tanto el significado matemático formal como el significado pragmático.

Además de eso, necesitaremos una noción de SMS lo suficientemente eficaz como para tratar con una teoría de Ia producción de SMS que incorpore los sistemas de signos intermediarios usados por el aprendiz en el proceso de enseñanza/aprendizaje-sistemas de signos intermediarios que el aprendiz tendrá que rectificar eventualmente, de manera que al final del proceso de enseñanza el estudiante llegue a ser competente.

Al ser tan idiosincráticos, algunos de estos sistemas de signos intermediarios no podrán ser considerados SMS, fundamentalmente por el carácter personal de los códigos inventados por el aprendiz, que no le permiten usar ese sistema de signos en un proceso de comunicación amplio debido a que éstos carecen de una convención 
socialmente acordada. Pero, como estamos tratando también con la observación de procesos de pensamiento matemático, tendremos que estar preparados para estudiar estos sistemas de signos con el fin de intentar intepretar los códigos personales del aprendiz. Esto es necesario para desvelar los obstáculos que produce la tensión de tratar con los SMS diferentes que el usuario tiene disponibles mientras está tratando de crear un nuevo SMS y de esa manera llegar a ser un "buen ejecutor" en los términos del significado pragmático socialmente determinado.

Cualquier modelo explicativo local teórico tiene que ocuparse de cuatro fuentes al menos de significado (Kaput 1987a):

1. Como resultado de las transformaciones en el interior de un SMS sin referencia a ningún otro SMS.

\section{Como resultado de las traducciones entre varios} SMS.

3. Como resultado de las traducciones entre SMS y sistemas de signos no matemáticos, tales como el lenguaje natural, imágenes visuales, y los sistemas de signos del comportamiento que usan los sujetos durante el proceso de enseñanza/aprendizaje. Los sistemas de signos del comportamiento nos permiten observar los procesos cognitivos de los aprendices y proponer, a partir de esos resultados psicológicos, nuevas hipótesis para un análisis didáctico matemático de los modelos de enseñanza implicados en el modelo teórico local bajo estudio.

4. Con la consolidación, simplificación, generalizacion y reificación de las acciones, procedimientos y conceptos de los SMS intermediarios creados durante las secuencias de enseñanza, esos SMS evolucionan hacia un nuevo SMS "más abstracto" en el que habrá acciones, procedimientos y conceptos nuevos que tendrán como referentes todas las acciones, procedimientos y conceptos de los SMS intermediarios para su uso en procesos de significacion nuevos. Si se alcanzan los objetivos del modelo de enseñanza, la nueva etapa tiene un nivel de organización más alto y representa una nueva etapa en el desarrollo cognitivo del aprendiz.

\section{Una teoría de la producción de SMS}

Mientras que las tres primeras fuentes de functores de signos (traducciones, según la terminología de Kaput) representan medios de tratar con expresiones primitivas y medios de combinarlas, la cuarta representa un medio de abstracción, gracias a la cual objetos compuestos pueden ser nombrados y manipulados como una unidad y, a continuación, ser usados en procesos de significación para resolver situaciones de resolución de problemas. Si, como así es en efecto, tenemos que trabajar con procesos de enseñanza/aprendizaje matemáticos, no hay manera de eludir el tener estos medios de abstracción como nuestro foco principal de observación. Así que necesitamos una teoría de la producción de SMS en la que un functor de abstracción relacione los diferentes SMS intermediarios (usados durante e1 desarrollo de las secuencias de enseñanza) con el SMS final más abstracto (el objetivo del modelo de enseñanza bajo estudio). Más adelante, un análisis didáctico matemático puede interpretar esta evidencia psicológica para proponer hipótesis relacionadas que sean observadas con sus propios medios metodológicos.

Exploraremos ahora la posibilidad teórica de un enfoque unificado de los fenómenos de significación matemática y/o de comunicación matemática que acabamos de describir. En la medida en que estamos en el inicio de este tipo de estudios, estamos condicionados por el estado actual de la investigación en educación matemática; estaremos explorando posibilidades teóricas de manera preliminar y posiblemente proponiendo cuestiones que, desde una perspectiva más avanzada, se dejarán de lado -lo que constituye uno de los supuestos metodológicos principales del uso de modelos teóricos locales, esto es, la inevitabilidad de su transformación (incluso de su abandono) cuando evidencia empírica ulterior hace necesario un marco teórico nuevo para interpretar problemas teóricos nuevos junto con una reinterpretación de los antiguos.

Llamaremos a un enfoque de este tipo una teoría semiótica del álgebra general, capaz de explicar los functores de signos matemáticos en términos de categorías de sistemas de signos subyacentes mutuamente correlacionados por uno o más códigos. Estas nociones tendrán que hacernos capaces de distinguir SMS de otros sistemas de signos y tendrán que comenzar la construcción de una noción de functor de abstracción de signos que pueda ser explicada en el interior de nuestra teoría de los códigos matemáticos.

Una teoría semiótica general de la matemática tendrá que considerar definiciones formales de cada tipo de functor entre sistemas matemáticos de signos, tanto si ha sido descrito ya al usuario o codificado por él, como si no lo ha sido. Así que la tipología de los modos de producción de SMS tendrá que proponer categorías capaces de describir incluso aquellas situaciones en las que están presentes functores de signos no codificados todavía (convencionalmente propuestos por el modelo de enseñanza) mientras son construidos por primera vez a través de un proceso de enseñanza.

\section{Observaciones finales}

En este artículo hemos resumido los temas principales de la investigación en álgebra en los últimos años. Hemos descrito también nuestros intentos de desarrollar una perspectiva teórica que nos permita encajar e interpretar los resultados empíricos existentes, y proponer nuevos diseños experimentales para hacer avanzar más la teoría. Un último asunto que nos gustaría tratar en este artículo tiene que ver con las areas de investigación en álgebra potencialmente fructíferas en el futuro.

Una de estas áreas es el desarrollo del pensamiento algebraico de los estudiantes. Unos pocos estudios han comenzado a abordar esta difícil área de investigación, 
pero queda mucho más por hacer. Uno de los problemas es la falta de acuerdo sobre lo que es exactamente el pensamiento algebraico. Ofrecemos, como un punto de partida, una breve caracterización hecha por Love hacia la que Wheeler (en prensa) atrajo nuestra atención:

"Hoy en día el álgebra no es meramente "dar significado a los símbolos" sino otro nivel más allá de eso; que tiene que ver con aquellos modos de pensamiento que son esencialmente algebraicos -por ejemplo, manejar Io todavía desconocido, invertir y deshacer operaciones, ver lo general en lo particular. Ser consciente de esos procesos, y controlarlos, es lo que significa pensar algebraicamente." [la cursiva es nuestra] (Love 1986, p. 49).

Otra área principal para la investigación futura es el papel de las computadoras en el aprendizaje de los conceptos algebraicos. La tecnología está remodelando nuestras nociones de lo que deberíamos enseñar y cómo deberíamos enseñarlo. El advenimiento de los manipuladores de símbolos, por ejemplo, sugiere que se podría gastar menos tiempo en aprender los aspectos manipulativos del álgebra y más en actividades que edifiquen la comprensión de conceptos algebraicos claves y habilidades de resolución de problemas. La dificultad obvia en el enfoque "menos destrezas" es evaluar el papel de la experiencia en procedimientos en el desarrollo de la comprensión por parte de los estu-

\section{REFERENCIAS BIBLIOGRÁFICAS}

ATHEN, H. y KUNLE, H. (eds.), 1976. Proceedings of the Third International Congress on Mathematical Education. (Universität Karlsruhe, Zentralbatt für Didaktik der Mathematik: Karlsruhe, RFA).

BAUERSFELD, H. y SKOWRONEK, H., 1976. Research related to the mathematical learning process, en Athen $y$ Kunle, (eds.), pp. 231-245.

BELL, A., LOW, B. y KILPATRICK, J., (eds.), 1984. Theory, Research \& Practice in Mathematical Education (Report of ICME5 Working Group on Research in Mathematics Education). (Shell Centre for Mathematics Education: Nottingham, UK).

BELL, A., O'BRIEN, D. y SHIU, C., 1980. Designing teaching in the light of research on understanding, en Karplus, (eds.), pp. 119-125.

BERGERON, J.C. y HERSCOVICS, N. (eds.), 1983. Proceedings of the Fifth Annual Meeting of PME-NA. (Université de Montréal: Montréal, Québec, Canada).

BERGERON, J.C., HERSCOVICS, N. y KIERAN, C. (eds.), 1987. Proceedings of the Eleventh International Conference for the Psychology of Mathematics Education. (Vol. I, II \& III). (Université de Montréal: Montréal, Québec, Canada). diantes de los conceptos algebraicos subyacentes. Como señala Fey (1987) "la interacción entre el conocimiento conceptual y procesual y el aprendizaje continuará siendo una cuestión absolutamente central sobre la que la investigación meditada puede aconsejar las decisiones curriculares" (p. 12).

En el pasado, la mayor parte de las matemáticas relacionadas con el álgebra se construían para usarlas en un medio estático. Pero ahora, con la llegada de las microcomputadoras, hay disponibles nuevos medios de uso que "cambian de modo fundamental las notaciones y las acciones que se usan para representar las relaciones y los procesos matemáticos" (Kaput 1987b, p. 30). La naturaleza dinámica del medio puede, en teoría, soportar conceptualizaciones de, por ejemplo, variable y función que son mucho menos accesibles en situaciones sin computadoras. La computadora permite un enfoque de la enseñanza del álgebra que pone el énfasis en los procesos y las acciones. Recordando los hallazgos de Sfard (1987) y otros al respecto del predominio significante entre los estudiantes de secundaria de las concepciones operacionales sobre las estructurales, ahora podemos, con la ayuda de las computadoras, desarrollar enfoques nuevos de la enseñanza del álgebra que están más en sintonía con una de las maneras de pensar y aprender álgebra preferidas por los estudiantes. Es un área rica en posibilidades de investigación.

BOOKER, G., 1987. Conceptual obstacles to the development of algebraic thinking, en Bergeron, J.C., Herscovics, N. y Kieran, C. (eds.), pp. 275-281.

BOOTH, L.R., 1981. Strategies and errors in generalised arithmetic, en Comiti y Vergnand, (eds.), pp. 140-146.

BOOTH, L.R., 1982. Developing a teaching module in beginning algebra, en Vermandel, (eds.), pp. 280-285.

BOOTH, L.R., 1983. A diagnostic teaching programme in elementary algebra: Results and implications, en Hershkowitz, (eds.), pp. 307-312.

BOOTH, L.R., 1984. Algebra: Children's strategies and errors. (NFER-Nelson: Windsor, UK).

BOOTH, L.R., 1987. Equations revisited, en Bergeron, J.C.; Herscovics, N. y Kieran, C., (eds.), pp. 282-288.

BROUSSEAU, G., 1986. Fondements et méthodes de la didactique des mathématiques, Recherches en Didactique des Mathématiques, Vol. 7, pp. 33-115.

CHAIKLIN, S. y LESGOLD, S., 1984. Prealgebra students' knowledge of algebraic tasks with aritmetic expressions. Paper presented at the annual meeting of AREA, New Orleans, LA. 
CHALOUH, L. y HERSCOVICS, N., 1984. From letter representing a hidden quantity to letter representing and unknown quantity, en Moser, (ed.), pp. 71-76.

CLEMENT, J., 1985. Misconceptions in graphing, en Streefland, (ed.), pp. 369-375.

COLLIS, K.F., 1975. The development of formal reasoning. (University of Newcastle: Newclastle, Austratia).

COMITI, C., y VERGNAUD, G., 1981. Proceedings of the Fifth International Conference for the Psychology of $M$ athematics Education. (Laboratoire I.M.A.G.: Grenoble, France).

COXFORD, A.F., (ed.), 1988. The ideas of algebra, $K-12$. 1988 Yearbook. (NCTM: Reston, VA).

DREYFUS, T. y EISENBERG, T., 1981. Function concepts: Intuitive baseline, en Comiti y Vergnaud, (eds.), pp. 183188.

DREYFUS, T. y EISENBERG, T., 1987. On the deep structure of functions, en Bergeron, J.C., Herscovics, N. y Kieran, C., (1987), Vol. I, pp. 190-196.

DUGDALE, S, 1982. Green Globs: A microcomputer application for graphing equations, Mathematics Teaching, Vol. 75, pp. 208-214.

ECO, U., 1979. A theory of semiotics. (Indiana University Press: Bloomington) [Trad. castellana, Tratado de semiótica general. (Lumen: Barcelona, 1981).].

FEY, J.T., 1989. School algebra for the year 2000, en Wagner y Kieran, pp. 199-213.

FILLOY, E., 1987. Modelling and the teaching of algebra, en Bergeron, J.C., Herscovis; N. y Kieran, C., (eds.), pp. 295300 .

FILLOY, E. y ROJANO, T., 1985a. Obstructions to the acquisition of elementary algebraic concepts and teaching strategies, en Streefland, (ed.), pp. 154-158.

FILLOY, E. y ROJANO, T., 1985b. From an arithmetical to an algebraic thought, en Moser, (ed.), pp. 51-56.

GALLARDO, A. y ROJANO, T., 1987. Common difficulties in the learning of algebra among children displaying low and medium pre-algebraic proficiency levels, en Bergeron, J.C.; Herscovics, N. y Kieran, C., (eds.), Vol, I, pp. 301-307.

GOLDENBERG, E.P., 1987. Believing is seeing: How preconceptions influence the perception of graphs, en Bergeron, J.C.; Herscovics, N. y Kieran, C., (ed.), Vol. I, pp. $197-203$.

GOLDIN, G., 1987. Cognitive representational systems for mathematical problem solving, en Janvier, (ed.), pp. 125145.

GREENO, J.G., 1982. A cognitive learning analysis of algebra. Paper presented at the annual meeting of AERA, Boston, MA.

HARPER, E.W., 1981. Psychological changes attending a transition from arithmetical to algebraic thought, en Comiti y Vergnaud, (eds.), pp. 171-176.

HART, K., (ed.), 1981. Children's understanding of mathematics: $11 \cdot 16$. (John Murray: London).

HERSCOVICS, N. y CHALOUH, L., 1984. Using literal symbols to represent hidden quantities, en Moser, (ed.), pp. 64-70.
HERSCOVICS, N. y KIERAN, C., 1980. Constructing meaning for the concept of equation, Mathematics Teacher, Vol. 73, pp. 572-580.

HERSHKOWITZ, R., (ed.), 1983. Proceedings of the Seventh International Conference for the Psychology of Mathematics Education. (Weizmann Institute of Science: Rehovot, Israel).

HOYLES, C., SUTHERLAND, R. y EVANS, J, 1985. The Logo Maths Project: A preliminary investigation of the pupil-centred approach to the learning of Logo in the secondary mathematics classroom, 1983-1984. (University of London, Institute of Education: London)

JANVIER, C., 1981. Difficulties related to the concept of variable presented graphically, en Comiti y Vergnaud, (eds.), pp. 189-192.

JANVIER, C., (ed.), 1987. Problems of representation in the teaching and learning of mathematics. (Lawrence Erlbaum: Hillsdale, NJ).

KAPUT, J.J., 1989. Linking representations in the symbol systems of algebra, en Wagner \& Kieran, (eds.), pp. $167-$ 194.

KARPLUS, R., (ed.), 1980. Proceedings of the Fourth International Conference for the Psychology of Mathematics Education. (University of California: Berkeley, CA).

KERSLAKE, D, 1977. The understanding of graphs, Mathematics in school, Vol. 6, pp. 22-25.

KIERAN, C., 1979. Children's operational thinking within the context of bracketing and the order of operations, en Tall, (ed.), pp. 128-133.

KIERAN, C., 1980. The interpretation of the equal sign: Symbol for an equivalence relation $v s$ an operator symbol, en Karplus, (ed.), pp. 163*169.

KIERAN, C., 1981. Concepts associated with the equality symbol, Educational Studies of Mathematics, Vol. 12, pp. 317-326.

KIERAN, C., 1983. Relationships between novices's views of algebraic letters and their use of symmetric and asymmetric equation-solving procedures, en Bergeron y Herscovics, (eds.) Vol. 1, pp. 161-168.

KIERAN, C., 1984. A comparison between novice and moreexpert algebra students on tasks dealing with the equivalence of equations, en Moser, (ed.), pp. 83-91.

KIERAN, C., 1985. Use of substitution procedure in learning algebraic equation-solving, en Shelton y Damarin, (eds.), pp. 145-152.

KIERAN, C., 1988. Two differents approaches among algebra learners, en Coxford, (ed.), pp. 91-96.

KIERAN, C., 1989. The early learning of aigebra: A structural perspective, en Wagner y Kieran, (eds.), pp. 33-56.

KIRSCHNER, D., 1987. The myth about binary representation in algebra, en Bergeron, J.C., Herscovics, N. y Kieran, C., (eds.), Vol. I, pp. 308-315.

KÜCHEMANN, D., 1981. Algebra, en Hart, (ed.), pp. 102119.

KUHN, T.S., 1962. The structure of scientific revolutions. (University of Chicago Press: Chicago) [Trad. castellana: La estructura de las revoluciones científicas (Fondo de Cultura Económica: México, 1971).]. 
LAPPAN, G. y EVEN, R., (eds.), 1986. Proceedings of the Eighth Annual Meeting of PME-NA. (Michigan State University: East Lansing).

LEE, L. y WHEELER, D., 1986. High school students' conception of justification in algebra, en Lappan y Even, (eds.), pp. 94-101.

LEWIS, C., 1980. Kinds of knowledge in algebra. Paper presented at the annual meeting of AERA.

LOVE, E., 1986. What is algebra?, Mathematics Teaching, 117, pp. $48-50$.

MARKOVITS, Z., EYLON, B.S. y BRUCKHEIMER, M., 1983. Functions-linearity unconstrained, en Hershkowitz, (ed.), pp. 271-277.

MATZ, M., 1980. Towards a computational theory of algebraic competence, Journal of mathematical behavior, Vol. 3, pp. 93-166.

MATZ, M., 1982. Towards a process model for high school algebra errors, en Sleeman y Brown, (eds.).

MERAVECH, Z.R. y YITSCHAK, D., 1983. Students' misconceptions of the equivalence relationship, en Hershkowitz, (eds.), pp. 313-318.

MOSER, J.M., (ed.), 1984. Proceedings of the Sixth Annual Meeting of PME-NA. (University of Wisconsin: Madison).

NESHER, P., 1980. The internal representation of open sentences in arithmetic, en Karplus, (ed.), pp. 271-278.

NUNES CARRAHER, T. y SCHLIEMANN, A.D., 1987. Manipulating equivalences in the market and in maths, en Bergeron, I.C., Herscovics, N. y Kieran, C., (eds.), Vol. I, pp. 289-294.

PETITTO, A., 1979, The role of formal and non-formal thinking in doing aigebra, Journal of Children's Mathematical Behavior, Vol. 2, pp. 69-82.

PONTE, I., 1985. Geometrical and numerical strategies in students' functional reasoning, en Streefland, (ed.), pp. $413-418$

PUTMAN, R.T., LESGOLG, S.B., RESNICK, L.B. y STERRET'T, S.G., 1987. Understanding sign change transformations, en Bergeron, J.C., Herscovics, N. y Kieran, C., (eds.), Vol. I, pp. 338-344.

ROGALSKI, J., 1985. Acquisition of number-space relationships: Using educational and research programs, en Streefland, (ed.), Vol. I, pp. 71-76.

SAMURCAY, R, 1985. Learning programming: Constructing the concept of variable by beginning students, en Streefland, (ed.), Vol. I, pp. 77-82.

SEIDEL, R.J., ANDERSON, R.E. Y HUNTER, B., (eds.), 1982. Computer literacy. (Academic Press: New York).

SFARD, A., 1987. Two conceptions of mathematical notions: Operational and structural, en Bergeron, I.C.; Herscovics, N. y Kieran, C., (eds.), Vol. III, pp. 162-169.

SHELTON, M. y DAMARIN, S., (eds.), 1985. Proceedings of the Seventh Annual Meeting of PME-NA. (Ohio State University: Columbus, Ohio).

SLEEMAN, D. y BROWN, J.S., (eds.), 1982. Intelligent tutoring systems. (Academic Press: New York).

SOLOWAY, E., LOCHHEAD, J. y CLEMENT, J., 1982. Does computer programming enhance problem solving abilify? Some positive evidence on algebra word problems, en Seidel, Anderson y Hunter, (eds.) .

STREEFLAND, L., (ed.), 1985. Proceedings of the Ninth International Conference for the Psychology of Mathematics Education. (State University of Utrecht: Utrecht, The Netherlands).

SUTHERLAND, R, 1987. A study of the use and understanding of algebra related concepts within a Logo environment, en Bergeron, J.C., Herscovics, N. y Kieran, C., (eds.), Vol. I, pp. 241-247.

SUTHERLAND, R. y HOYLES, C., 1986. Logo as a context for learning about variable, en Proceedings of the Tenth International Conference for the Psychology of Mathema. tics, pp. 301-306. (University of London, Institute of Education: London, U.K.)

TALL, D., 1987. Constructing the concept image of a tangent, en Bergeron, J.C.; Herscovics, N. y Kieran, C., (eds.), Vol. I, pp. 69-75.

TALL, D., (ed.), 1979. Proceedings of the Tenth International Conference for the Psychology of Mathematics Education, pp. 313-318. (University of Warwick: Coventry, U.K.).

THOMAS, M. y TALL, D., 1986. The value of the computer in learning algebra concepts, en Proceedings of the Tenth International Conference for the Psychology of Mathematics Education, pp. 313-318. (University of London, Institute of Education: London, U.K.).

THOMPSON, P.W. y THOMPSON, A.G., 1987. Computer presentations of structure in algebra, en Bergeron, J.C.; Herscovics, N. y Kieran, C., (eds.), Vol, I, pp. 248-254.

VERGNAUD, G., 1984. Understanding mathematics at the secondary-school level, en Bell, Low y Kilpatrick, (eds.), pp. $27-35$.

VERGNAUD, G. y CORTÉS, A., 1986. Introducing algebra to "low-level" eight and ninth graders, en Proceedings of the Tenth International Conference for the Psychology of Mathematics Education, pp. 319-324. (University of London, Institute of Education: London, U.K.).

VERGNAUD, G. y ERRECALDE, O., 1980. Some steps in the understanding and the use of scales and axis by $10-13$ years old students, en Karplus, (ed.), pp. 285-291.

VERMANDEL, A., (ed.), 1982. Proceedings of the Sixth International Conference for the Psychology of Mathematics Education. (Universitaire Instelling Antwerpen: Antwerp, Belgium).

WAGNER, S. y KIERAN, C., eds., 1989. Research issues in the learning and teaching of algebra. (NCTM y Lawrence ErIbaum: Reston, VA y Hillsdale, $N I)$.

WAGNER, S., RACHLIN, S.L. y JENSEN, R.J., 1984. Algebra learning project: Final report. (University of Georgia, Department of Mathematics: Athens).

WHEELER, D., 1989. Context for research on the teaching and learning of algebra, en Wagner y Kieran, (eds.), pp. 278-287.

ZEHAVI, N. 1986. Interaction between graphical and algebraic representations in the use of microcoumputer software, en Proceedings of the Tenth International Conference for the Psychology of Mathematics Education, pp. 217-222. (University of London, Institute of Education: London, U.K.). 